\title{
Einige Experimente zur Entwicklungsmechanik der Amphibienhörbläschen.
}

\author{
Von \\ Chikanosuke Ogawa. \\ Anatomisches Institut der Kaiserlichen Universität zu Kyoto.
}

Mit 6 Textfiguren.

Entwicklungsmechanische Untersuchungen beschränkten sich anfangs hauptsächlich nur auf sehr frühe Entwicklungsstadien, wie das Stadium der Eizelle oder das der Furchungszelle. Aber mit der Zeit sind bei der experimentellen Forschung auch spätere Stadien herangezogen worden. Das Augenmerk der Entwicklungsmechaniker hat sich so nach und nach den inneren Faktoren der Entwicklung zugewendet.

Besonders dürften sich meines Erachtens für diese Forschung Organe eignen, welche erstens leicht zugänglich, zweitens nicht lebenswichtig und drittens frühzeitig differenzierbar sind. Am besten erfüllen diese Bedingungen die Sinnesorgane, und so liegen betreffs des Sehorgans bereits verhältnismässig zahlreichen Arbeiten vor, unter denen besonders eingehend das Linsenregenerationsproblem behandelt worden ist. Dagegen finden sich auf dem Gebiete des Gehörorgans nur die Publikationen von Streeter, Spemann, Lewis, Levy, Tokura, Sternberg und mir. Das mag daran liegen, dass das Gehörorgan gegen den mechanischen Angriff nur eine ganz träge Gestaltungsreaktion aufweist und experimentell nicht sehr verheissend erscheint. 'Trotzdem aber habe ich meine Experimente fortgesetzt und will sie im Folgenden mit den erzielten Resultaten kurz wiedergeben.

Als Materialien wählte ich Bufo japonicus, Rana nigromaculata, und Diemictylus pyrrhogaster. Die Operationen wurden in Kochsalzlösung vorgenommen, die ich darauf allmählich durch Leitungswasser ersetzte.

\section{Inversion des Hörbläschens.}

Streeter hat zuerst gezeigt, dass, wenn man das Hörbläschen der Anurenlarve im non-motilen Stadium nach Hautschnitt von seiner 
Umgebung ablöst und sein Innen nach aussen kehrt, ohme dabei auf oben und unten Rücksicht zu nehmen, das Bläschen in der weiteren Entwicklung in seine normale Lage zurückkehrt. Spemann hat dagegen gezeigt, dass sich das Hörbläschen der Larve, nachdem man sein Oben und Unten vertauscht und dabei in bezug auf die Lage innen und aussen nicht verändert hat, in der abnormen umgekehrten Lage weiter entwickelt.

Meine Nachprüfung bestätigte diese Resultate, worüber ich in meiner vorigen Arbeit berichtet habe. Betreffs der Meinungen über die Verschiedenheit der Ergebnisse verweise ich auf Tokura's Arbeit.

Ich stellte die folgenden Experimente in der Hauptsache nach Streeter's Methode an.

a. Inversion des halbierten Hörbläschens.

Wie schon oben angeführt, steht fest, dass ein Hörbläschen die. Fähigkeit besitzt, in seine alte Lage zurückzukehren, wenn innen und aussen mit einander vertauscht werden. Es ist nun interessant zu erfahren, ob dieses Vermögen nur dem kompletten Hörbläschen innewohnt, oder ob auch einem Teil eines Bläschens diese Fähigkeit eigen ist. Um darüber Gewissheit zu erhalten, habe ich das folgende Experiment ausgeführt.

Die Operation an Bufo wurde am Ende des non-motilen Stadiums vorgenommen. Bei dem für die Operation günstigsten Stadium scheint es sich nur um eine sehr beschränkte Zeit zu handeln, indem die Operation in scheinbar demselben Stadium nicht immer gleich leicht gelingt. Die Ohranlage ist in dem Stadium schon bläschenförmig. Man geht folgendermassen vor: Nach seiner Blosslegung wird das Hörbläschen an der unteren Hälfte zwischen zwei Nadeln gequetscht und die dadurch locker gewordene Masse abgerissen. Dabei wurden die Nadeln mit drei Fingern so gehalten, wie wir Orientalen es mit den Essstäbchen tun. Auch lässt sich für die Operation die Uhrmacherpinzette gebrauchen. Man reponiert nun die zurückgebliebene obere Partie des Hörbläschens, vertauscht innen und aussen, wobei man darauf Rücksicht nimmt, dass der obere Pol des Bläschens abwärts und die untere offene Seite auf- und etwas zerebralwärts gerichtet werden, um zu verhindern, dass die Wunde des Hörbläschens und die der Haut miteinander verwachsen. Bei allen Fällen war der Ductus endolymphaticus noch nicht deutlich zur Ausbildung gelangt.

Der Grund, dass die obere Hälfte des Bläschens zurückgelassen wurde, war, dass später der Saccus und der Duetus endolymphaticus daraus 
hervorgehen und mit Vorteil als Anhaltepunkt für die Orientierung benutzt werden können.

Ich operierte im ganzen 17 'Tiere, wovon eins nach 5 Tagen, der Rest nach 4 Wochen getötet wurde.

Beschreibung der einzelnen Fälle :

Bufo A 4 A. 5 Tage post operat.: In die normale Lage zurückgekehrt.

Hörbläschen klein. Die Bogengänge noch nicht gebildet, Ductus endolymphaticus liegt normal. Am ventralen Teil des Bläschens hängt eine unregelmässig zusammengeballte Ganglienmasse.

Bufo.A 4 B. 4 Wochen post operat. : In die normale Lage zurückgekehrt.

Ohrlabyrinth klein und mit der Knorpelkapsel umgeben, Saccus endolymphaticus, Lagena und Macula neglecta in normaler Lage, Bogengänge noch nicht vorhanden, während sie auf der normalen Seite schon in Ausbildung begriffen sind. Ganglion acusticum vorhanden.

Bufo A 4 a. 4 Wochen post operat. : In die normale Lage zurückgekehrt.

An der Wandung des Labyrinthes haben sich kurze Septen gebildet, Bildung der Bogengänge ist ausgeblieben, Saccus endolymphaticus und Lagena liegen normal, Ganglion acusticum klein und mit dem Gehirn nicht verbunden.

Bufo A 4 b. 4 Wochen post operat. : In die normale Lage zurückgekehrt.

Ductus endolymphaticus und Lagena sind nachweisbar, der laterale Bogengang hat sich gebildet und ist mit der Knorpelkapsel umgeben. Ganglion acusticum ist vorhanden und mit dem Gehirn verbunden. Doch ist die Lage nicht ganz typisch.

Bufo A 4 c. 4 Wochen post operat. : Schwer zu entscheiden, ob die neue Iage die normale ist.

Septumbildung mangelhaft, Bogengänge nicht entstanden, ein saccusartiges Gebilde liegt ventrolateralwärts, Lagena nicht nachweisbar, Ganglion nicht erkennbar.

Bufo A 4 d. 4 Wochen post operat. : Vielleicht in die normale Lage zurückgekehrt.

Der laterale Bogengang hat sich gebildet, ein dünnwandiges Bläschen ist am ventromedialen Teil ausserhalb der Knorpelkapsel vorhanden, Kommunikation mit dem Labyrinth nicht nachweisbar. Herstammung dieses Bläschens nicht klar. Lagena fast in normaler Lage, Ganglion acusticum nicht vorhanden. 
Bufo A 4 e. 4 Wochen post operat. : In die normale Lage zurückgekehrt (Fig. 1).

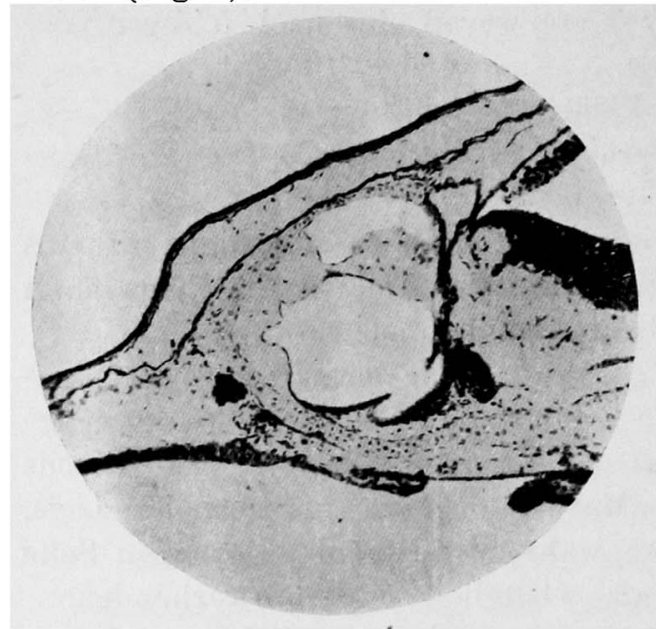

Fig. 1.

Bufo japonicus. Das in die normale Lage zurückgekehrte Ohrlabyrinth. 4 Wochen nach Halbierung und Umkehrung. Ductus endolymphaticus in normaler Lage, $\times 50$.

Labyrinth klein, Saccus und Lagena in normaler Lage vorhanden, Bogengänge teilweise gebildet, Ganglion acusticum mit dem Gehirn verbunden.

Bufo B $4 \mathrm{f}$. 4 Wochen post. operat.

Hörbläschen nicht vorhanden.

Bufo A 4 g. 4 Wochen post. operat.: In die normale Lage nicht zurückgekehrt.

Labyrinth klein, Septum so gut wie nicht entwickelt, Saccus. endolymphaticus abnorm gestaltet und dorsal liegend, Ductus mit dem Labyrinth am lateralen 'Teil verbunden. Macula neglecta liegt ventrolateralwärts. Ganglion accisticum nicht vorhanden.

Bufo A $4 \mathrm{~h}$. 4 Wochen post operat.: Wahrscheinlich in die normale Lage zurückgekehrt.

Ductus nicht vorhanden, Ganglion acusticum klein, in normaler Lage und mit dem Gehirn verbunden. Lage des Labyrinthes schwer festzustellen.

Bufo A 4 i. 4 Wochen post operat. : In die normale Lage zurückgekehrt.

Labyrinth ziemlich gut gebildet. Oberer und hinterer Bogengang ausgebildet, Saccus und Lagena in normaler Lage. Ganglion acusticum. mit dem Gehirn verbunden.

Bufo A $4 \mathrm{j}$. 4 Wochen post operat.

Labyrinth nicht vorhanden.

Bufo A 4 k. 4 Wochen post operat.: In die normale Lage zurückgekehrt.

Ductus endolymphaticus hat sich aus dem medialhinteren Teil der Labyrinthhöhle entwickelt, Saccus medial in der Knorpelkapsel verborgen, also mit dem Gehirn nicht in Berührung gekommen, teilweise jedoch frei aus der Knorpelkapsel hervortretend. Lagena in normaler 
Einige Experimente zur Entwicklungsmechanik der Amphibienhörbläschen.

Lage vorhanden. Ganglion acusticum klein und mit dem Gehirn verbunden.

Bufo A 4 l. 4 Wochen post operat. : In die normale Lage zurückgekehrt.

Bogengänge unvollständig ausgebildet, Saccus und Lagena in normaler Lage. Ganglion acusticum mit dem Gehirn verbunden.

Bufo A $4 \mathrm{~m}$. 4 Wochen post operat.: In die normale Lage zurückgekehrt.

Labyrinth klein, dickwandiger Saccus vorhanden, Bogengänge nicht gebildet. Ganglion acusticum klein und mit dem Gehirn nicht verbunden.

Bufo A $4 \mathrm{n}$. 4 Wochen post operat.: In die normale Lage zurückgekehrt.

Bogengänge nicht gebildet, Saccus endolymphaticus liegt medial doch innerhalb der Knorpelkapsel. Lagena in fast normaler Lage. Ganglion acusticum klein, mit dem Gehirn nicht verbunden.

Bufo A 4 o. 4 Wochen post operat.: Rückkehr in die normale Lage nicht sicher entscheidbar.

Labyrinth klein, Bogengänge nicht gebildet, Saccus vorhanden. Lagena liegt abwärts, Ganglion acusticum nicht sicher nachweisbar.

Wie dieses Protokoll zeigt, war in 2 Fällen kein Hörbläschen zu sehen. Es ist wahrscheinlich nach der Operation aus der Schnittwunde geschlüpft. Unter den übrigen 15 Fällen ist das Ohrlabyrinth in einem Fall nicht, in 12 Fällen sicher in die normale Lage zurückgekehrt, während bei 2 Fällen die Lagebestimmung ummöglich war. Danach ist in den meisten Fällen das Hörbläschen in die normale Lage zurückgekehrt. Der Saccus endolymphaticus ist meistenfalls vorhanden und leistet gute Dienste bei der Lagebestimmung, zu der auch Lagena und Ganglion acusticum beigetragen.

Es ist ohne weiteres klar, dass das operierte Hörbläschen in allgemeinen mangelhaft differenziert war. Während an der normalen Seite die Differenzierung ziemlich fortgeschritten ist, hat sich auf der operierten Seite nur ein einzelner Bogengang oder gar keiner gebildet. In der Mehrzahl der Fälle findet man jedoch den Saccus endolymphaticus ausgebildet und in normaler Lage vor. Danach dürfte er bei der Operation nicht gelitten haben. Trotz mangelhafter Bildung der Bogengänge ist die Lagena fast immer zur Ausbildung gelangt. Aus dieser Tatsache darf man schliessen, dass die Lagena in der Entwicklung sehr beständig, mindestens weit bestündiger als der Bogengang ist. Man kann eigentlich nicht gut begreifen, dass sich die Lagena, welche norma- 
lerweise im ventroposterioren Abschnitt des Labyrinthes liegt, ausgebildet hat, obgleich das Hörbläschen an seiner unteren Hälfte gequetscht worden ist. Doch gibt es hierfür zwei Erklärungsmöglichkeiten. Erstens könnte bei der Operation mehr der lateral-untere Abschnitt des Hörbläschens abgetragen worden sein. - Tatsächlich ist bej einer technisch so schweren Operation wie dieser keine mathematische Genauigkeit zu erwarten. Zweitens mag die untere Hälfte zwar abgetragen worden sein, aber die nachher entstandene untere Wandung hat von neuem die Fähigkeit erlangt, die Lagena neu zu bilden.

Gegen diese zweite Möglichkeit spricht Streeter's Anschauung, nach der das Hörbläschen frühzeitig differenziert ist und kein Regenerationsvermögen besitzt. Da sich das Labyrinth nun nach Abtragung der unteren Hälfte zum geschlossenen Bläschen umbildet, besitzt es sicher ein gewisses Reparationsvermögen. Aber es ist nicht leicht denkbar, dass das Bläschen so regulatorisch ist, dass die geschlossene untere Wandung die Lagena ausbildet.

In allen Fällen, gleichgültig ob die Differenzierung fortgeschritten ist oder nicht, ist das Ohrlabyrinth von der Knorpelkapsel umgeben. Bei normaler Entwicklung liegt gewöhnlich der Saccus endolymphaticus immer ausserhalb der Knorpelkapsel dicht am Gehiru. Zwei operierte Fälle (n, k) zeigen jedoch, dass der Saccus teilweise innerhalb der Knorpelkapsel zur Entwicklung gelangt ist, während seine Lage (Saccus) nicht ganz normal ist. Wie unten auseinandergesetyt wird, ist die Knorpelkapsel wahrscheinlich durch Anregung von seiten des Ohrlabyrinthes aus dem umgebenden Mesenchym entstanden. Die Tatsache, dass bei normaler Entwicklung eine Knorpelkapsel in der Umgebung des Saccus endolymphaticus fehlt, spricht dafür, dass der Saccus das umgebende Mesenchym nicht zur Knorpelbildung anregt. Bei meinen Fällen ist jedoch die Knorpelkapsel in der Umgebung des Saccus vorhanden. Das mag daher kommen, dass der Saccus hier noch nicht vollständig differenziert war und die Eigenschaft als allgemeine Labyrinthwandung beibehalten hatte.

Jedenfalls ist bewiesen, dass das invertierte Hörblïschen, wenn es auch halbiert wurde, in seine normale Lage zurückkehren kann.

b. Inversion des Hörbläschens in mehr fortgeschrittenem Stadium.

Für das Inversionsexperiment kam bisher nur das non-motile Stadium der Amphibienlarve in Betracht. Es bedarf nun noch weiterer Untersuchung, ob das Hörbläschen auch im fortgeschrittenen Stadium die Fähigkeit, in die normale Lage zurückżukehren, besitzt oder nicht. 
Für die Operation wurden Bufolarven von 8-9 mm Körperlänge (16-17 Tage nach dem Verlassen des Eies) gebraucht, bei welchen die äusseren Kiemen schon ausgebildet und die Gegend der Anlage der vorderen Extremitäten vorgetrieben waren. Die Larven machten ab und zu rudernde Bewegungen. Man führt die Operation unter Chloroformnarkose aus. Die laterale Wandung des Hörbläschens ist nach dem Hautschnitt aus der Haut leicht abtrennbar. Am oberen Abschnitt dagegen ist der Ductus endolymphaticus schon ausgebildet und wegen seiner ziemlich festen Verbindung mit der Unterlage von dieser nicht leicht abzulösen. Da nach der Abtragung des Hörbläschens die Mesenchymmasse immer der medialen Wandung anhaftet, läuft man nicht Gefahr, die Seiten zu verwechseln.

Das Hörbläschen ist bald birnförmig, bald relativ kuglig, prall gefüllt und für die Manipulationen fest genug.

Die Operation wurde an 18 Exemplaren ausgeführt. Da die Fixation bald- 3 oder 4 Tage- nach der Operation stattfindet, ist die Differenzierung des Labyrinthes noch nicht fortgeschritten. Die Orientierung wurde nach der Lage des Ductus endolymphaticus beurteilt. Auch die Macula wurde dazu herangezogen. Das Ganglion acusticum ist gewöhnlich nicht vorhanden. In anderen nicht veröffentlichten Versuchsreihen mit Ranalarven bemühte ich mich, das Ganglion acusticum aus der Hörbläschenwandung $\mathrm{zu}$ beseitigen. Leider hatte ich fast stets Misserfolge zu verzeichnen. Aber bei den jetzigèn Versuchen konnte ich das Ganglion ohne besondere Manipulationen entfernen, wovon die Ursache möglicherweise darin zu suchen ist, dass diesmal bei der Operation das Entwicklungsstadium schon weiter fortgeschritten und die Verbindung zwischen dem Ganglion und dem Hörbläschen demgemäss locker geworden war.

Die Beurteilung der Lage fand nach denselben Gesichtspunkten wie beim vorigen Experiment statt. Darum verzichte ich hier auf die Beschreibung der einzelnen Fälle und gebe nur kurz die Resultate an.

Unter 18 Exemplaren ist das Hörbläschen in zwei Fällen ausgeschlüpft. Bei den übrigen 16 Exemplaren zeigte sich Folgendes:

Rotiert (in die normale Lage zurückgekehrt) 7 (Fig. 2).

Partiell rotiert 2.

Schwer entscheidbar 4.

Nicht rotiert 3 (Fig. 3). 




Fig. 2 .

Rana nigromaculata. Das in der normalen Lage stehen gebliebene Hörbläschen. 4 Tage nach dem Umkehrungsversuch im fortgeschrittenen Stadium, $\times 50$.

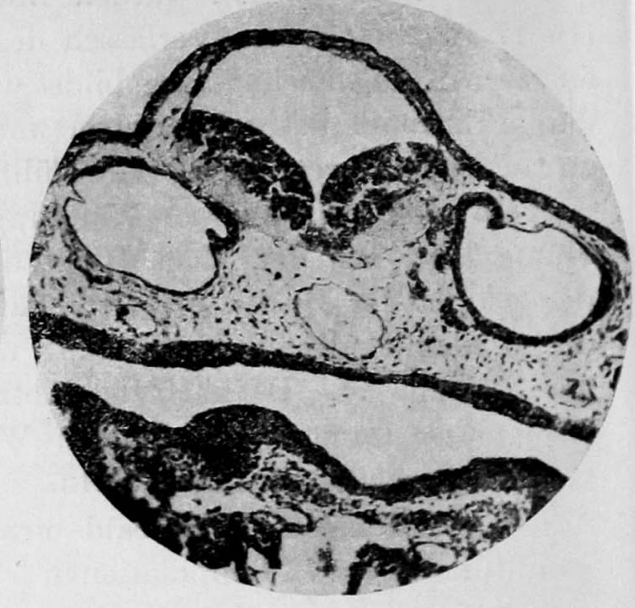

Fig. 3.

Rana nigromaculata. Das in die normale Lage zurückgekehrte Hörbläschen. $う$ Tage nach dem Umkehrungsversuch im fortgeschrittenen Stadium, $\times 50$

Wenn man hierbei die rotierten und die nicht rotierten Hörbläschen miteinander vergleicht, lässt sich in bezug auf das Verhalten des umgebenden Gewebes zum Bläschen keine besondere Ursache, wie zu wenig Raum u. s. w., für das Ausbleiben der Rotation ausfinding machen. Danach scheint die Nichtrotation ihrem Wesen nach nicht rein mechanisch zu sein.

Die obigen Versuche zeigen klar, dass das Hörbläschen auch im mehr fortgeschrittenen Stadium (Körperlänge $8-9 \mathrm{~mm}$, äussere Kiemen schon ausgebildet) die Rückrotationsfähigkeit besitzt. Während aber im non-motilen Stadium fast alle Hörbläschen zurückzurotieren imstande sind, besitzt in diesem Stadium nur ungefähr die Hälfte diese Fähigkeit. Dieser Befund zeigt, dass die Rückrotationsfähigkeit des Hörbläschens mit fortschreitender Entwicklung allmählich abnimmt.

Wie verhält sich nun das Hörbläschen, wenn die Operation in noch späterem Stadium vorgenommen wird? Vom technischen Standpunkt aus ist jedoch diese Operation fast unmöglich, weil in den späteren Stadien das Hörbläschen schon zu weit gewachsen und nur noch ein spärlicher Spaltraum in der Umgebung übrig und es mit dieser zu fest verwachsen ist. 
c. Inversion des Hörbläschens, welches von einem jungen Individuum auf ein älteres transplantiert worden ist.

Für die durch das vorige Experiment klargelegte Tatsache, dass sich der Prozentsatz der Rückrotation des Hörbläschens im fortgeschrittenen Stadium etwas vermindert, möchte ich annahmsweise zwei ursächliche Momente verantwortlich machen: Entweder ist das umgebende Gewebe älter geworden, oder das Hörbläschen hat sich zu weit entwickelt.

Wenn das letztere richtig wäre, müsste die Rückrotation leichter vor sich gehen, wenn ein jüngeres Hörbläschen auf ein älteres Individuum in umgekehrter Lage transplantiert würde.

Das folgende Experiment wurde ausgeführt, um diese Frage zu beantworten.

Als Fortsetzung des vorigen Experimentes wäre es sicher besser géwesen, dieselbe Spezies für den Versuch zu gebrauchen. Aber da diese Spezies wegen der späten Jahreszeit nicht mehr erhältlich war, bediente ich mich der Rana nigromaculata. Die jüngere Larve befand sich wie gewöhnlich im non-motilen Stadium; die ältere Larve besass zwei geteilte Kiemenstummel, einen deutlich ausgebildeten Schwanz und zeigte $a b$ und zu eigene Bewegung, besonders bei Berührung mit der Nadel. Der Ductus endolymphaticus war deutlich ausgebildet, so dass das Hörbläschen Birnform darbot.

Obwohl das Stadium der Larve dem im Experiment b nicht genau gleich war, machte das praktisch nicht weiter viel aus. Ich operierte zuerst an jungen Fröschen und danı an älteren. Im ganzen wurden 8 Paar Exemplare operiert und nach 4 Wochen fixiert.

Ohne mich auf eine detaillierte Beschreibung einzulassen, will ich hier sofort kurz das Resultat mitteilen.

An zwei Exemplaren sucht man vergebens nach dem Hörbläschen. Die übrigen 6 Exemplare zeigen in der Mehrzahl

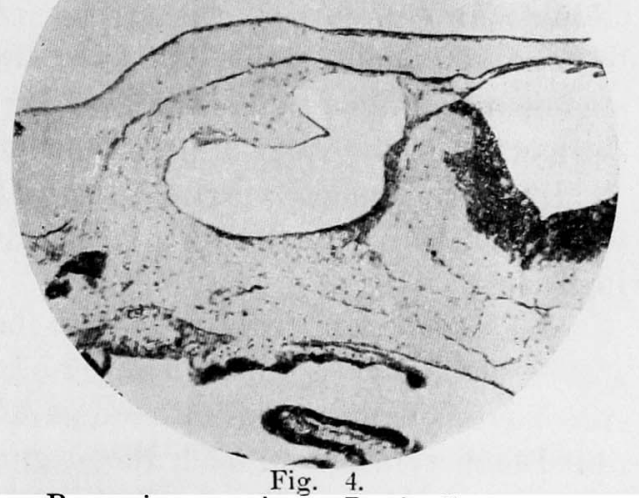

Rana nigromaculata. Das in die normale Lage zurückgekehrte Hörbläschen, das als jüngeres Hörbläschen in umgekehrter Lage auf eine ältere Larve transplantiert wurde. 4 Wochen nach der Transplantation, $\times 50$. 
den Ductus endolymphaticus und differenzierte Bogengänge, wodurch man leicht entscheiden kann, dass die Hörbläschen alle zurückrotiert sind (Fig. 4).

Danach ist auch ein auf ein älteres Individuum transplantiertes Hörbläschen der Rückrotation fähig. Im vorigen Experiment waren die Hörbläschen nur zur Hälfte rückrotationsfähig. Dafür kann man als Ursache zwei Möglichkeiten annehmen : Entweder war das umgebende Gewebe oder das Hörbläschen schon zu weit entwickelt. Mittels des Resultates des jetzigen Experimentes lässt sich erklären, warum im vorigen Experiment die Hörbläschen nur teilweise rotationsfähig waren. Die betreffenden nicht rotierten Hörbläschen befanden sich nämlich in fortgeschrittenem Stadium, sodass die Versuchsreihen nur zum Teil erfolgreich ausfielen, während das Stadium des umgebenden Gewebes dafür ohne Bedeutung war.

Um diesen Schluss noch zwingender zu gestalten, wäre es nötig, ein altes Hörbläschen auf ein jüngeres Individuum zu transplantieren. Da aber das Hörbläschen im Verhältnis zum Wirt relativ gross ist, ist die Transplantation leider technisch unausführbar.

\section{Zerstörung des Hörbläschens.}

Bei normaler Entwicklung bildet sich die Knorpelkapsel um das Ohrlabyrinth, wenn die Differenzierung des letzteren oder anders ausgedrückt der ganze Organismus einen gewissen Entwicklungsgrad erreicht hat. Dabei ist schon von manchen Seiten (Lew is, Spemann) bewiesen worden, dass das Ohrlabyrinth das umgebende Mesenchym zur Knorpelbildung anregt. Aber wie verhält es sich dann, wenn die zelligen Bestandteile nicht mehr das Labyrinth, sondern nur einen unregelmässigen Klumpen bilden? Wird der nicht differenzierte, ordnungslose Zellhaufen seine Umgebung obenso wie der differenzierte beeinflussen ?

Das vorliegende Experiment sollte hier Klarheit bringen. Leider ist es jedoch völlig misslungen. Das Resultat ist aber anderweitig von Bedeutung.

Als Versuchstier verwendete ich im ersten Jahr Dicmictylus pyrrhogaster und Rana nigromaculata, im nächsten Rana nigromaculata allein. Alle Tiere befanden sich im non-motilen Stadium. Zuerst stach ich an fünf Diemictyluslarven nach Blosslegung des Hörbläschens dieses mehrmals mit der Haarnadel an. Dadurch glaubte ich, dass sich das Bläschen in ein ganz lose zusammenhängendes Zellklümpchen verwandelt habe. Nach 4 Wochen mikroskopierte ich und fand, dass sich die Hörbläschen alle normal entwickelt hatten. Ich schrieb diesen Misserfolg unge- 
nügender Zerstörung des Hörbläschens zu und wiederholte im folgenden Jahre dieselbe Operation, indem ich diesmal bei Rana nach partieller Heilung der Hautwunde, die zwecks sicherer Orientierung gesetzt worden

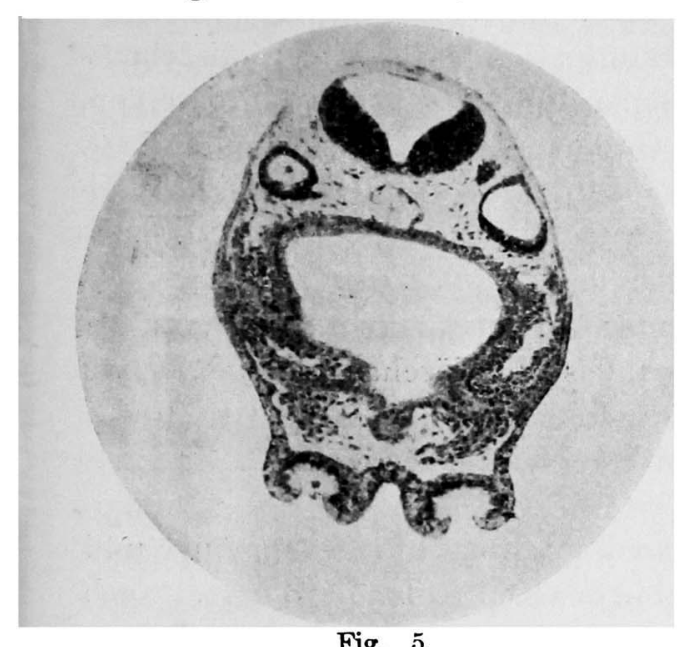

Fig. 5 .

Rana nigromaculata. Das Hörbläschen 20 Stunden nach der Zerstörung (links), $\times 50$. war, an verschiedenen Stellen das Hörbläschen stark anstach. Im ganzen wurden 16 Exemplare operiert. Als Kontrolle wurden nach 20 Stunden 4 Exemplare fixiert. Obwohl es scheinbar zweckmässiger ist, die Fixation sofort nach der Operation auszuführen, ist das doch in der Tat unmöglich, da die lose zusammenhängende, augestochene Masse in der Fixierungsflüssigkeit leicht aus der offenen $W$ unde entweichen würde.

Die übrigen 12 Larven wurden alle nach einem Monate

oder 4 Wochen fixiert.

Auch jetzt stellte sich heraus, dass sich alle Hörbläschen wieder zu Labyrinthen von fast normaler Gestalt entwickelt hatten. Sellst an der Kontrolle boten die Hörblaschen normales Aussehen dar (Fig. 5). Da die erstmalige Operation erfolglos, war ich bei der zweiten Operation sehr energisch vorgegangen, und doch erhielt ich wieder fast normale Ohrlabyrinthe. Das lässt sich nur so erklären, dass sich das durch Anstechung zertrümmerte Hörbläschen wieder zu einem normalen Labyrinth ausbildet. Danach besitzt das Hörbläschen eine hochgradige Reparationsfähigkeit.

\section{Elimination des Hörbläschens.}

Es ist üblich, ein Organ oder einen Organteil zu beseitigen, um Kompensation, Regeneration oder Ausfall der Funktion zu studieren. Das jetzige Experiment bezweckt aber, die direkte lokale Veränderung zu verfolgen, welche durch Elimination des Hörbläschens in der Nachbarschaft stattfindet.

Ein Organ nimmt in einer bestimmten Region bestimmte Form und Gestalt an, damit zwischen dem Organ und der Umgebung das Gleichgewicht erhalten bleibt. Wenn also ein Organ eliminiert wird, müsste 
man aus den in der Umgebung stattgefundenen Veränderunge» die "topographische Funktion" dieses Organs erschliessen können. Ich glaube, dass für derartige Forschungen die Bezeichuung „, experimentelle Topographie " passend wäre und dabei als Methodik hauptsächlich Elimination in Frage käme. Die Elimination kann weder an lebenswichtigen Organen noch an erwachsenen Individuen ausgeführt werden, weil im letzteren Falle die Lage des Organs meistenfalls schon fixiert ist.

Also dürfte die experimentelle Topographie als ein Gebiet der experimentellen Embryologie von Bedeutung sein.

Ich eliminierte an 39 Bufolarven das Hörbläschen einseitig. Die Operation geschah ungefähr am Ende des non-motilen Stadiums: die Kiemengegend war etwas vorgewölbt, das Hörbläschen bläschenförmig und durch Operation leicht zu öffnen. Die Wunde blieb nach der Operation offen, und die Mesenchymzellen flossen nicht aus.

Das Gehörorgan liegt an der Seite des Gehirns hinter dem Auge und bildet einen wesentlichen Bestandteil in der Kopfregion der Larve. Nach Elimination dieses Organs wird. wie später angegeben, die entstandene Lücke durch das Mesenchymgewebe ersetzt. Die äussere Form des Kopfes erhält sich also mehr oder weniger symmetrisch, obwohl hierbei das Munddach an der eliminierten Seite höher steht.

Als direkter Einfluss der Elimination des Gehörorgans will ich noch die Lageveränderung des Augapfels erwähnen. Bei der Besichtigung der nach 14 und 23 Tagen fixierten Präparate befindet sich der Augapfel in ganz normaler Lage und zeigt keine wesentliche Verschiebung. Daher ist in diesem Stadium die Laye des Bulbus unabhängig von der Existenz des Ohrlabyrinthes. Das ist leicht zu verstehen, wenn man die Lage beider Organe berücksichtigt, da ja das Hörorgan $200 \mu$ hinter dem Bulbus liegt. An den nach 7 bis 10 Wochen fixierten Exemplaren beobachtet man, dass allmählich eine kaudale Verschiebung des Bulbus stattfindet. Danach ist die Lage des Bulbus in diesem Stadium abhängig vom Gehörorgan, und beide Organe müssen unter gegenseitigem Druck in ihrer Lage verbleiben.

Es ist oben schon erwähnt worden, dass sich die Knorpelkapsel des Ohrlabyrinthes durch Anregung von seiten dieses letzteren Organs bildet. Wenn das Ohrlabyrinth eliminiert wird, bleibt im wesentlichen die Knorpelbildung in der Region aus. Aber nach genauer Überlegung zeigt sich, dass die Verhältnisse nicht so einfach liegen. Der mediale Abschnitt der Knorpelkapsel bildet z.B. gleichzeitig de laterale Wand des Schädels. Es muss daher entschieden werden, ob die Knorpel- 
bildung hier von der Existenz des Hörbläschens abbängig ist oder nicht. Die Basalplatte setzt sich lateralwärts noch der unteren und medialen Abteilung der Knorpelkapsel fort. Es kann nur experimentell nachgewiesen werden, wie gross hierbei das vom Ohrlabyrinth abhängige Gebiet ist.

Ich habe daher im Folgenden einzelne Fülle genau beschrieben.

Bufo Al C A. 2 Wochen post operat.

In diesem Stadium ist die Basalplatte der prïchurdalen Region von der Ohrkapsel getrennt. In der chordalen Region ist der laterale Bogengang von der dünnen Knorpelkapsel umgeben. Auf der eliminierten Seite findet man keine Knorpelkapsel. Auf der normalen Seite geht die Parachordalia, sich allmählich verjüngend, in die untere Abteilung der Ohrkapsel über, wührend : ie auf der Seite mit Elimination kurz ist und in der lateralen Schädelwand in der Höhe des Ganglion acusticum verschwindet. In diesem Stadium ist also die Bildung der Basalplatte und der Fo tsetzung derselben in die Chordalregion abhängig vom Ohrlabyrinth in dem Gebiet ausserhalb der durch den medialen Rand des Ganglion acusticum gezogenen Sagittalelene.

Bufo Al C B. 2 Wochen post operat.

Betreffs der Basalplatte liegen die Verhältnisse wie beim vorigen Fall. Auf der Seite mit Elimination ist das Palatoquadratum ungefähr um $120 \mu$ länger als auf der normalen. Da die Austrittstellen der Hirnnerven beiderseits symmetrisch in dem Schnitt anzutreffen sind, so ist. ohne weiteres klar, dass hierbei die Schnittrichtung richtig war. Es sei jedoch dahingestellt, ob diese Verlängerung ganz zufällig oder von derElimination abhängig ist. Man findet in der Ohrregion ein mit einfachem, kubischem Epithel bekleidetes rundliches Bläschen, welches von einer dünnen Knorpelkapsel umgeben ist. Es muss sicher aus dem bei der Elimination zurückgebliebenen Rest entstanden sein.

Bufo Al C C. 2 Wochen p. op.

Nichts Besonderes.

Bufo Al C A. 23 Tage p. op.

Bufo Al C B. 23 Tage p. op.

Im grossen und ganzen wie die vorigen Fälle.

Bufo Al C A. 5 Wochen p. op.

In diesem Stadium ist auf der normalen Seite die Entstehung einer dünnen Knorpelkapsel zwischen Gehirn und Ohrlabyrinth bemerkbar. Auf der Seite mit Elimination ist das laterale Ende der Parabasalplatte etwas nach oben gekrümmt, und es fehlt die eigentliche Knorpelkapsel. Obwohl die mediale Abteilung der Ohrkapsel gerade die laterale Schädei- 
wand bildet, fehlt doch hier die Knorpelbildung, wenn das Hörbläschen nicht vorhanden ist.

Bufo Al C B. 5 Wochen p. op.

Elimination des Hörbläschens misslang.

Bufo Al C C. 5 Wochen p. op.

Bufo Al C D. 5 Wochen p. op.

Keine Knorpelkapsel.

Bufo Al C A. 7 Wochen p. op.

Auf der normalen Seite sieht man die Knorpelbildung in der medialen, hinteren Abteilung der Ohrkapsel, während auf der Seite mit Elimination keine Knorpelbildung nachweisbar ist. Auf der Seite mit Elimination ist der Bulbus etwas nach hinten gelagert und das Palatoquadratum auf dieser Seite um $180 \mu$ länger als auf der normalen.

Bufo Al C B. 7 Wochen p. op.

Keine Knorpelbildung auf der Seite mit Elimination.

Bufo Al C C. 7 Wochen p. op.

A uf der Seite mit Elimination findet man schwache Knorpelbildung kaudal vom N. facialis, u. zwar ist das Schädeldach etwas nach unten und die Basalplatte etwas nach oben ungebogen, jedoch ohne Vereinigung beider Knorpelenden.

Bufo Al C D. 7 Wochen p. op.

In der Prächordalregion der Seite mit Elimination stösst man am oberen Teil der seitlichen Schädelwand auf zirkumskripte Knorpelbildung.

Bufo Al C E. 7 Wochen p. op.

Keine Knorpelbildung auf der Seite mit Elimination.

Bufo Al C F. 7 Wochen p. op. (Fig 6).

Auf der Seite mit Elimination findet sich ein aus einfachem plattem Epithel entstandenes kleines Bläschen, welches von einer Knorpelkapsel umgeben ist. Die Kapsel steht lateral-

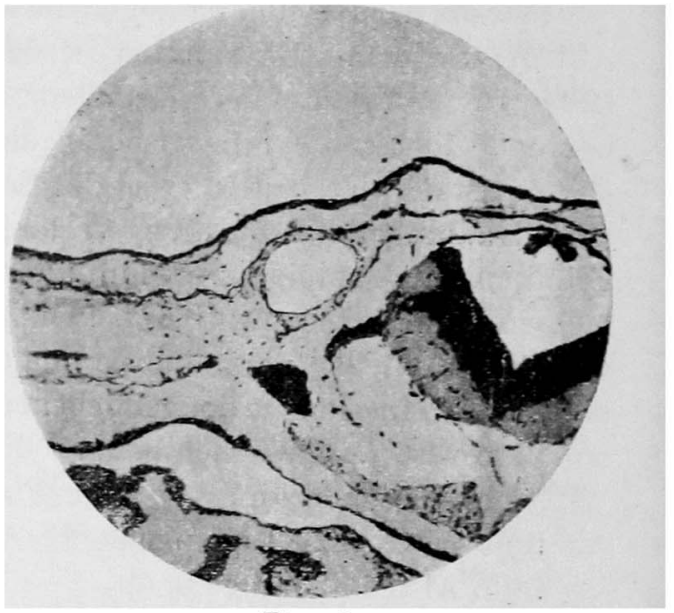

Fig. 6.

Rana nigromaculata. Das einfache Bläschen, welches aus der bei der Elimination des Hörbläschens zurückgebliebenen Zellmasse entstanden and mit der Knorpelkapsel umgeben ist. 7 Wochen nach der Operation, $\times 50$. wärts direkt mit der Pigmentschicht der Haut in Berührung. Sonst ist keine Knorpelbildung zu sehen. 
Bufo Al C G. 7 Wochen p. op.

Auf der Seite mit Elimination ist keine Knorpelbildung zu sehen.

Bufo Al C H. 7 Wochen p. op.

Auf der Seite mit Elimination ist das laterale Ende der Basalplatte etwas aufwärts gekrümmt.

Bufo Al C I. 7 Wochen p. op.

Gleich wie $\mathrm{H}$.

Bufo Al C J. 7 Wochen p. op.

Auf der Seite mit Elimination ist keine Knorpelbildung zu sehen.

Bufo Al C A. 8 Wochen p. op.

Auf der Seite mit Elimination ist keine Knorpelbildung zu sehen.

Bufo Al C B. 8 Wochen p. op.

Auf der Seite mit Elimination ist ein schwach differenziertes Bläschen vorhanden, welches mit einer ziemlich dicken Knorpelkapsel umgeben ist.

Bufo Al C C. 8 Wochen p. op.

Auf der Seite mit Elimination ist ein grosses, von einer Knorpelkapsel umgebenes Bläschen vorhanden.

Bifo Al C D. 8 Wochen p. op.

In der chordalen Region der Seite mit Elimination findet man eine dünne Knorpelplatte in der lateralen Wandung des Schädels, welche mit der Basalplatte in keiner Verbindung steht.

Bufo Al C E. 8 Wochen p. op.

Wie oben.

Bufo Al C F. 8 Wochen p. op.

Auf der Seite mit Elimination hat sich in der lateralen Wandung des Schädels eine schwache Knorpelplatte gebildet, welche mit dem Schädeldach sowie der Basalplatte verbunden ist.

Bufo Al C G. 8 Wochen p. op.

In der chordalen Gegend ist ein schwaches Knorpelstück in der lateralen Wandung des Schädels vorhanden.

Bufo Al C H. 8 Wochen p. op.

Fast wie G.

Bufo Al C I. 8 Wochen p. op.

Das Ohrlabyrinth ist der Elimination entgangen.

Bufo Al C J. 8 Wochen p. op.

Auf der Seite mit Elimination keine Knorpelbildung zu konstatieren.

Bufo Al b A. 10 Wochen p. op.

Auf der lateralen Seite des Schädels der Seite mit Elimination ist schwache Knorpelbildung nachweisbar. 
Bufo Al b F. 10 Wochen p. op.

Fast wie A.

Bufo Al b G. 10 Wochen p. op.

Auf der Seite mit Elimination ist das laterale Ende der Basalplatte aufwärts gebogen und bildet die laterale Wand des Schädels.

Bufo Al b H. 10 Wochen p. op.

Auf der Seite mit Elimination ist nichts von Knorpelbildung zu sehen.

Aus diesen Befunden darf man schliessen, dass keine Knorpelkapsel gebildet wird, wenn in der Ohrregion das Labyrinth fehlt. Auch andere Autoren haben das schon nachgewiesen. Die mediale Abteilung der Ohrkapsel bildet aber zugleich die laterale Wand des Schädels. Es muss hier zunächst entschieden werden, ob die Bildung des Knorpels an dieser Stelle vom Ohr oder vom Hirn abhängig ist. An den Präparaten des früheren Stadiums vermisst man auf der Seite mit Elimination Knorpelbildung in der laterale Schädelwand, während sie auf der normalen Seite schon stattgefunden hat. In einem etwas späteren Stadium stösst man dort auf ein kleines Knorpelstück, welches jedoch für die Bildung der Schïdelwand zu schwach ist. Danach ist das Vorhandensein des Ohres für die Bildung der lateralen Schädelwand notwendig, wenigstens in dem von mir untersuchten Stadium. Das Ohrlabyrinth regt also die Bilung der lateralen Schädelwand an. Die 'Tatsache, dass in einem etwas späteren Stadium eine schwache ungenügende Knorpelbildung nachweibar ist, möchte ich als eine Regulationserscheinung auffussen. Im folgenden Jahre führte ich noch einmal dasselbe Experiment aus und bestätigte nach Abschluss dieser Arbeit, dass in noch älteren Stadien die Knorpelbildung der Schädelwand auf der Seite mit Elimination vollständig ist.

Die Basalplatte geht normalerweise lateralwärts in die Ohrkapsel über. In diesem Falle ist klar zu stellen, wie weit sich die Basalplatte nach der Elimination des Hörbläschens bzw. nach dem Unterbleiben der Bildung der Knorpelkapsel lateralwärts ausdehnt. Die Resultate des Experimentes zeigen, dass die Basalplatte in den meisten Fällen lateralwärts mit plötzlicher Verjüngung an der Sagittalebene endet, welche die dem Sitze des Ganglion acusticum entsprechende Stelle passiert. Daür dürfte es nicht weiter gewagt erscheinen, normalerweise als die laterale Grenze der Basalplatte die Sagittalebene des Ganglion acusticum zu setzen. Zuweilen zeigt die Basalplatte an der lateralen Seite des Gehirns eine leichte Biegung nach oben.

Bei normaler Entwicklung bildet sich die Ohrkapsel immer, nach- 
dem die Differenzierung des Hörbläschens einen gewissen Grad erreicht hat. Aber bei meinem Experimente zeigte sich, dass das einfache Blïschen, welches sicher aus dem Reste der ungenügend eliminierten Anlage entstand, von der Knorpelkapsel umgeben war. Ich hatte einige solche Fïlle. Danach ist klar, dass selbst ein äusserlich undifferenziertes Hörbläschen das umgébende Mesenchym zur Knorpelbildung anregen kann. Betreffs der Bedingungen der Knorpelbildung, sind hierbei nach genauerer Überlegung drei Momente möglich. Erstens : Wenn das Bläschen auch nach Gestalt und Bau einfach erscheint, können doch die zelligen Bestandteile ganz wie in :ilteren Stadien bereits im wesentlichen differenziert sein und auf das umgebende Mesenchymgewebe knorpelbildend wirken. Zweitens kann der ganze Organismus bzw. das Mesenchymgewebe einen gewissen Entwicklungsgrad erreicht haben, bei dem durch Anregung von seiten des undifferenzierten Hörbläschens die Knorpelbildung stattfindet. Drittens kann der Ohrknorpel durch das Zusammenwirken der oben angegebenen zwei Momente zustande kommen. Es bedarf daher weiterer Experimente, um zu entscheiden, welche dieser drei Möglichkeiten bei der Knorpelbildung die Hauptrolle spielt. Ich möchte vorläufig das zweite Moment als die Ursache betrachten.

\section{Transplantation des Hörbläschens hinter das normale.}

Von den Transplantationsversuchen, welche zum Nachweise der Rückrotationsfähigkeit ausgeführt worden sind, war schon die Rede. Hier möchte ich cinige gemachte Experimente kurz besprechen, durch die ermittelt werden sollte, in welchem gegenseitigen Verhältnis hintereinander gelagerte-ein normales und ein transplantiertes-Hörbläschen ihre Entwicklung fortsetzen. Ein ähnlicher Versuch ist schon von Streeter ausgeführt worden. Daher handelt es sich bei dem meinigen mehr um eine Nachprüfung.

Die Exemplare wurden im non-motilen Stadium operiert. Ein aus einem anderen Tiere herausgenommenes Hörbläschen wurde dicht hinter das gleichseitige normale transplantiert, indem dabei die Lagebeziehung des hinteren Hörbläschens zur Körperachse möglichst normal erhalten wurde. Bei den 6 zu diesem Versuche verwendeten Tieren geschah die Fixation nach drei Wochen.

Ein Exemplar ging zugrunde, ein anderes beherbergte kein transplantiertes Hörbläschen. Bei den übrigen gediehen die transplantierten Gebilde aufs beste. Der Kürze halber verzichte ich hier auf Einzelbeschreibung. 
Das vordere Labyrinth entwickelte sich in fast normaler Weise, während an dem hinteren transplantierten die Bogenänge ungenügend differenziert waren. Beide Labyrinthe berührten sich dicht mit einander und verschmolzen gewöhnlich mit den sich berührenden Wandungen, wobei zuweilen zwischen beiden Labyrinthhöhlen eine Verbindung vorhanden war. Das nach Entfernung des normalen Hörbläschens transplantierte Hörblüschen entwickelte sich, wie schon von manchen Seiten gezeigt, fast normal ohne erhebliche Abnormititen. Woher kommt es nun, dass sich in diesem Falle das transplantierte Labyrinth allein abnorm entwickelt? Zunächst muss man daran denken, dass zwei Hörbläschen dicht beisammen liegen und so auf einander einen Druck ausübeis. Würe aber der Druck die Trsache der abnormen Entwicklung der transplantierten Hörblïschen, dann müsste sich des Druckes wegen auch das normale Hörbläschen abnorm entwickeln. Man kann sich nicht gut vorstellen, dass sich durch gegenseitigen Bruck ein Hörbläschen normal, das andere abnorm entwickelt. Zweitens ist die Lage in Rücksicht zu ziehen. Das vordere Hörbläschen liegt normal, während des hintere eine, wenn auch nur geringe abweichende Position einnimmt. Sieht man von dem gegenseitigen Druck als Ursache der Abnormität ab, so bleibt nur die abnorme Lage als Ursache übrig.

Das Ganglion acusticum ist bald vorhanden, b ld nicht, wenn vorhanilen, so sendet es seine Nervenfasern wach den Neuroepithelzellen, während es mit dem Gehirn nicht in Verbindung tritt.

\section{Ergebnisse.}

1. Wenn das Hörbläschen an der unteren Hälfte abgerissen und bei der zurückgebliebenen oberen Hälfte innen und aussen miteinander vertauscht werden, kann es in der weiteren Entwicklung wieder normale Lage einnehmen. In diesem Punkte verhält sich das halbierte Hörblïschen ganz gleich wie das vollständige.

2. Der Inversionsversuch des Hörbläschens wurde anfangs nur an ganz jungen Amphibienlarven und zwar etwa am Ende des non-motilen Stadiums vorgenommen. Ich invertierte innen und aussen des Hörbläschens im fortgeschrittenen Stad.um und zwar an der Bufolarve von 8-9mm, bei der die äusseren Kiemen schon ausgebildet und die vorderen Extremitätenanlagen vorgetrieben waren. In der Hälfte der Fälle fand sich das Hörbläschen in die normale Lage zurückrotiert. Danach ist das Hörbläschen auch in etwas fortgeschrittenem Stadium rückrotationsfähig. Doch hat im Vergleich mit dem jüngeren Stadium die Fähigkeit stark abgenommen. 
3. Wenn man bei dem Hörbläschen einer jüngeren Ranalarre im non-motilen Stadium innen und aussen miteinander vertauscht und das Bläschen auf eine ïltere Larve transplantiert, welche sich im Stadium der Zweiteilung der Kiemenstummel und deutlicher Schwanzbildung befindet, so rotiert das Hörblïschen in die normale Lage zurück. Für das nur teilweise Gelingen der Rückrotation beim vorigen Versuch im älteren Stadium darf man deshalb die Ursache nur im fortgeschrittenen Entwicklungszustande des Hörblïschens selbst und nicht im umgebenden Gewebe suchen.

4. Zerstört man durch tiefes Einstechen das Hörbläschen der Diemictylus- und Ranalarve im non-motilen Stadium, so heilt es im weiteren Verlauf wieder zu einem normalen Labyrinth zusammen. Danach zeigt das Hörblïschen Reparationsvermögen, indem es, wie bekannt, keiner Regeneration fähig ist.

5. Nach Elimination des Hörbläschens bleibt die Bildung der Knorpelkapsel aus. Dabei sieht man auch an der lateralen Schädelwand der Ohrregion keine Knorpelbildung, wonach also diese Knorpelbildung vom Ohr abhängig ist. In einem etwas fortgeschritteneren Stadium bemerkt man jedoch daselbst eine Knorpelbildung, welche als nachherige Regulationserscheinung aufgefasst werden kann. Die Basalplatte bildet sich unabhängig vom Ohrlabyrinth lateralwïrts bis zur Sagittalebene des Ganglion acusticum.

6. Die Knorpelkapsel kann sich um das einfache Bläschen bilden, welches aus dem Rest des nicht genügend eliminierten Hörbläschens entstanden ist.

7. Bei der Transplantation des Hörbläschens hinter das normale entwickelt sich das transplantierte abnorm, und es wird dabei zuweilen Verschmelzung der Wandungen beider Labyrinthe beobachtet.

\section{Literatur.}

1. Ogawa, Experiments on the orientation of the ear vesicle in amphibian larves. Journ. of exper. Zool. Vol. 34. 1921.

2. Spemann, Die Entwicklung des invertierten Hörgrübchens zum Labyrinth. Arch. f. Entw.-Mech. Bd. 30. 1910.

3. Sternberg, Ueber Transplantation des Hörbläschens bei Froschlarven. Arch. f. mikr. Anat., Bd. 103.1924.

4. Streeter, Some experiments on the developing ear vesicle of tadpole with relation to equilibration. Journ. of exper. Zool. Vol. 3. 1906.

5. - - Some factors in the development of the amphibian ear vesicle and further experiments on equilibration. Journ. of exper. Zool. Vol. 4. 1907.

6. - - Experimental evidence concerning the determination of posture of the membranous labyrinth in amphibian embryos. Journ. of exper. Zool. Vol. 16. 1914.

7. Tokura, Zur Frage der Hörbläscheninversion. Folia anat. Jap. Bd. 2. 1924. 